Scientific Paper

\title{
Calibration of Gafchromic XR-RV3 film under interventional radiology conditions
}

\author{
Joanna KIDON' ${ }^{a,}{ }^{*}$, Kinga POLACZEK-GRELIK ${ }^{b}$, Krzysztof GOŁBAc, Wojciech WOJAKOWSKI ${ }^{d}$, Andrzej OchAŁA ${ }^{a}$ \\ ${ }^{a}$ Invasive Cardiology and Electrocardiology Department, 3rd Department of Cardiology, Medical University of Silesia, \\ Katowice, Poland \\ ${ }^{b}$ Prof. K. Gibiński Memorial University Clinical Centre, Medical University of Silesia, Poland \\ ${ }^{c}$ Department of Electrocardiology and Heart Failure, Medical University of Silesia, Poland \\ ${ }^{d} 3 \mathrm{rd}$ Department of Cardiology, School of Medicine in Katowice, Medical University of Silesia, Poland \\ *E-mail address: joanna.kidon@sum.edu.pl
}

\begin{abstract}
Introduction: The purpose of the study was the calibration of Gafchromic films in clinical interventional radiology conditions and the assessment of the influence of dose range, the shape of the fitting curve, and its practical application. The aim of the work was to show how practically perform calibration in a wide range of doses.

Material and methods: Gafchromic XR-RV3 films were included in the study. The calibration was performed for A and B film series separately. Doses from the range of $0-8$ Gy were used. Film dosimeters were read out in reflective mode with a commercial flatbed scanner.

Results: Among various degrees of a polynomial function, the best fit, which fulfilled the chosen criterion of $95 \%$ agreement between measured and reconstructed doses and simple equation criterion, was observed for third-degree polynomial. The fitting curve where the dose is the function of optical density (logMPV) was demonstrated to be more precise than the fitting curve based on MPV only. To minimize the difference between dose absorbed by the film and dose reconstructed from the fitting curve below $5 \%$ it is necessary to divide the calibration range of $0-8$ Gy into two subranges for use in interventional radiology. This difference was set at a maximum level of 3.8\% and 1.9\% for the lowand high-dose range, respectively. Each series of films may have a slightly different calibration curve, especially for the low dose range. A deviation of up to $36 \%$ between two batches of Gafchromic film was observed.

Conclusions: For the third-degree polynomial fitting function (one of the recommended in the literature) calibration should be done into low and high dose ranges and for each batch separately. A systematic error higher than $20 \%$ could be introduced when the fitting curve from one film batch is applied to the other film batch.
\end{abstract}

Key words: calibration curve; Gafchromic film; XR-RV3; fluoroscopically guided intervention.

\section{Introduction}

The evaluation of radiation doses received by patients in cardiological procedures is important for several reasons. Interventional procedures using an X-ray unit are high-dose procedures that can lead to radiation damage to the patient's skin. Moreover, dose metrics, which are routinely recorded during the procedure, do not constitute the peak skin dose (PSD) on the patient's skin, because they are an indicator of the tube output and cannot be readily used for estimating the patient skin dose. ${ }^{1,2}$ PSD is responsible for the appearance of the deterministic effects of ionizing radiation. One of these indicators is kerma at the reference point $\left(\mathrm{K}_{\mathrm{air}, \mathrm{ref}}\right)$, as defined by the IEC 60601-2-43 standard. This dosimetric parameter is most suitable for determining PSD, but it corresponds to PSD only in strictly defined conditions. $\mathrm{K}_{\text {air,ref }}$ is not equal to the patient dose in most clinical cases.
The verification of doses received by patients undergoing interventional radiology procedures is a time-consuming process depending on some factors (low dose rate compare to radiotherapeutic units to establish a calibration curve, the need for passive dosimeter processing, and data analysis). ${ }^{2-4}$ The basis for this is the use of appropriate dosimetry systems and their proper calibration. Among the methods used for dose measurement in clinical practice, radiochromic films are commonly used in radiation therapy for dosimetric verification of treatment plans, ${ }^{5-7,8}$ whereas in interventional radiology, they are used to assess the surface dose on the patient's skin(peak skin dose ). ${ }^{3,9}$ This kind of film is also used for instance to improve CT acquisition image processes ${ }^{10}$ and to check the distributions of the laser-accelerated proton beams. ${ }^{11}$ Since this dosimetric method is a relative one, the proper calibration curve needs to be determined prior to its use for dose assessment. ${ }^{2,3,4,10-14}$ In the

(C) 2021 Joanna Kidon, Kinga Polaczek-Grelik, Krzysztof Gołba, Wojciech Wojakowski, Andrzej Ochała. This is an open access article licensed under the Creative Commons Attribution-NonCommercial-NoDerivs License (http://creativecommons.org/licenses/by-nc-nd/4.0/). 
literature there is still an insufficient number of detailed descriptions of the calibration curve fitting analysis for XR-RV3 films. Due to the relatively low dose rate of ionizing radiation of $\mathrm{X}$-ray units used in interventional radiology compared to radiotherapeutic units, this process performed in clinical conditions is somewhat time-consuming. Therefore, film detectors might be calibrated in laboratory conditions, or calibration strips dedicated to a given series of films could be used. Laboratory conditions and those in which calibration strips have been exposed may differ from clinical conditions in terms of the quality of the ionizing radiation beam. Therefore, in order to maintain the high precision of the reconstruction of a dose received by the patient, calibration of films in clinical conditions is recommended. ${ }^{4}$

Dosimetric Gafchromic XR-RV3 type films are easy to use in the clinical setting, since they do not interfere with $\mathrm{x}$-ray parameters or image quality, do not cause discomfort to the patient, are insensitive to visible light, and are tissue equivalent. ${ }^{4}$ These films are self-developing and provide 2D dose mapping. ${ }^{2,15,16}$ To obtain dose information, however, it is necessary to read them, i.e. to quantify the optical density changed as a result of dose deposition, using a scanner and determine the calibration curve. The dosimetric properties of these films also depend on their homogeneity, the energy of ionizing radiation, storage conditions, and sensitivity to UV light. ${ }^{4}$ According to the film manufacturer, in the dose rate range from $0.03 \mathrm{~Gy} / \mathrm{min}$ to $3 \mathrm{~Gy} / \mathrm{min}$ the dependence of the film response from the dose rate is below 3\%. ${ }^{4}$ Therefore it is believed that the dose rate variation does not affect the calibration process of the films. ${ }^{17}$ The detailed technical aspects of six Gafchromic film types are described by Devic et al. ${ }^{18}$ However, this description did not include the XR-RV3 type, although this work contains thoroughly discussed recommendations during the calibration process, which were also taken into account in the presented study.

The purpose of the study was to check the influence of dose ranges taken into account in this process, the shape of the fitting curve, and its practical application on the process of film dosimeter calibration). The choice of the appropriate calibration curve is also discussed $(D=f(M P V)$ versus $D=f(\log M P V)$, where D - dose, MPV - mean pixel value or application of a calibration curve from a different package of films). The aim of the work was not to find a new fitting function than recommended in the literature, but to show how to practically deal with the calibration in a wide range of doses.

\section{Materials and Methods}

\section{XR-RV3 Gafchromic films}

XR-RV3-type Gafchromic films from Ashland Inc. were used in the calibration process. The properties of the films, according to the manufacturer's data (personal communication), are the high spatial resolution $(\leq 25 \mu \mathrm{m})$, high contrast, with a recommended range of application from $0.2 \mathrm{~Gy}$ to $10 \mathrm{~Gy}$ and from about $30 \mathrm{keV}$ to $30 \mathrm{MeV}$, and dose rate independence. The general characteristics of XR - RV3 films used during measurements are presented in Table 1.

XR-RV3 Gafchromic films, commonly used in interventional radiology, are read (scanned) in the reflection mode. These films consist of four layers: a yellow/orange polyester layer $(97 \mu \mathrm{m})$, an adhesive layer made of acrylic $(20 \mu \mathrm{m})$, an active layer $(17 \mu \mathrm{m})$, and a white polyester layer $(97 \mu \mathrm{m}) .{ }^{19}$ The layer sensitive (active) to the ionizing radiation is lithium salt of pentacosa-10,12-diynoic acid $\quad$ (LiPDCA). ${ }^{19} \quad$ XR-RV3 Gafchromic films are tissue-equivalent dosimetric systems, because the effective $\mathrm{Z}$ of all of these layers is approximately 7.3. ${ }^{20}$ As a result of ionizing radiation interaction, free radicals are formed, which undergo stabilizing polymerization at least 24 hours after exposure. ${ }^{19}$ According to the information obtained from the film manufacturer, the polymerization process begins within $100 \mu \mathrm{s}$ from the end of the exposure. Below $100 \mathrm{~Gy}$, polymerization progresses as a rapid (within about $30 \mathrm{~ms}$ ) firstdegree reaction, which transforms within a second into the slow phase, in which the changes in optical density are proportional to the logarithm of time after exposure. A significant part of the polymerization process (about $90 \%$ ) occurs within the first four hours after irradiation, reaching approximately $99 \%$ after 48 hours (personal communication).

\section{Calibration process}

Taking into account the dependence of film optical density after irradiation on the quality of the X-ray beam, fluoroscopy mode (continuous, pulsed), and the discrepancy between the optical density of films irradiated in clinical and laboratory conditions (up to $15 \%),{ }^{4}$ the calibration in the presented study was performed in clinical conditions, separately for A and B film series, since the optical properties of each film batch may vary significantly.

All films were stored in accordance with the manufacturer's instructions, i.e. temperature indicator on the control bar as recommended, and no access to sunlight and UV light. During exposure to ionizing radiation, the film was placed with a sensitive (orange) layer toward the $\mathrm{X}$-ray tube.

Table 1. Basic data of XR-RV3 Gafchromic films, based on data from certificates provided with these dosimeters.

\begin{tabular}{cccccc}
\hline \hline Batch & Date of manufacture & Expiration date & Sensitivity [du] & Density before exposure [du] & Nonuniformity [\%] \\
\hline A & $24.08 .2018 \mathrm{r}$. & 24.03 .2020 & $1.19(\geq 0.95)$ & $0.20(\leq 0.40)$ & $2.01(<7)$ \\
B & $02.01 .2019 \mathrm{r}$. & 02.07 .2020 & $1.17(\geq 0.95)$ & $0.20(\leq 0.40)$ & $1.71(<7)$ \\
\hline \hline
\end{tabular}

allowable values are given in brackets, $d u$ - density units 


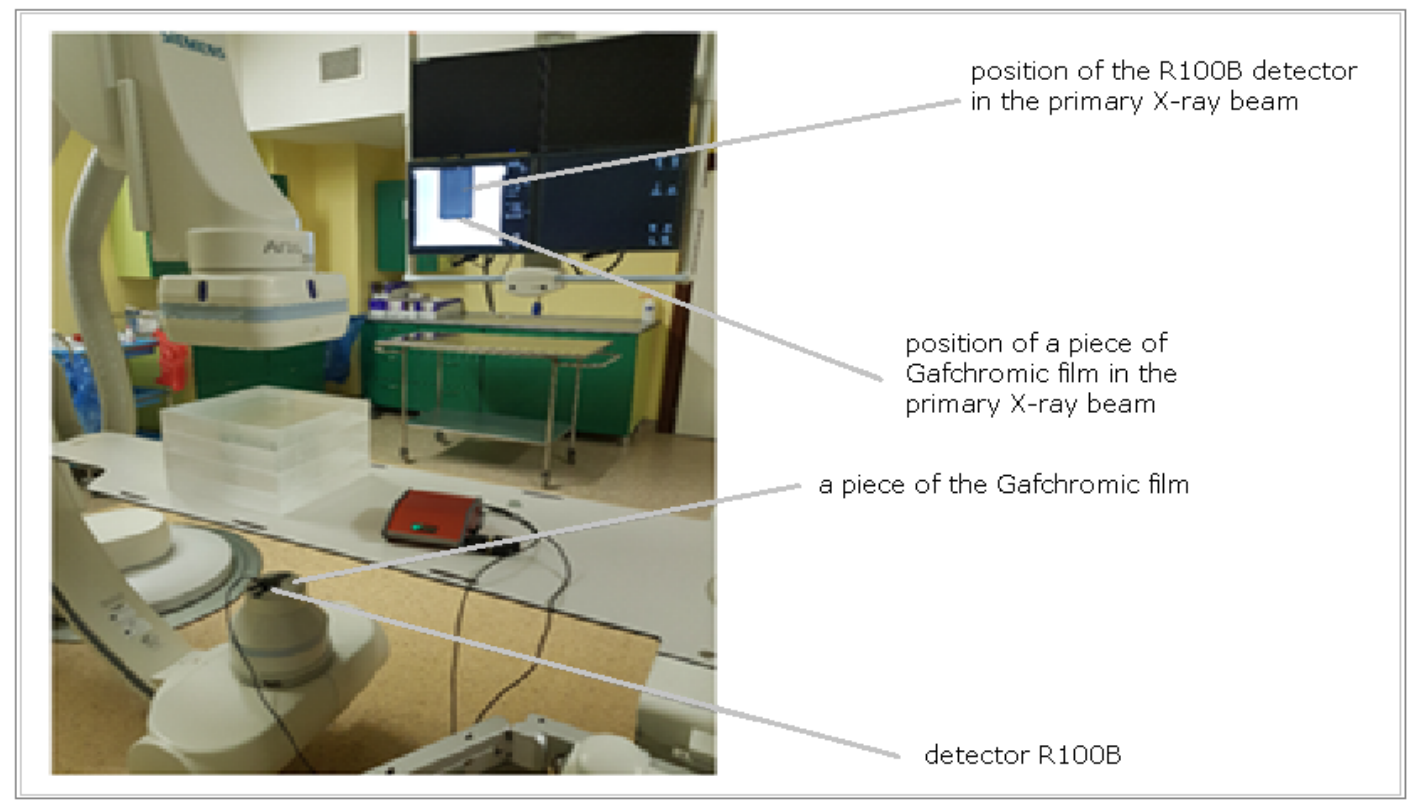

Figure 1. Measuring system - calibration of Gafchromic films.

Each film was marked with a film production serial number. At least 48 hours after the irradiation, films were scanned with an Optice Pro A320 scanner (reading in a reflective mode, color recording, 24 bits, resolution $300 \mathrm{dpi}$, without compression or postprocessing and other image modifications, recorded in BMP format). Prior to the film scanning, the scanner was automatically heated up. Scanning direction perpendicular to the long axis of the film was used for all films. The mean pixel values (MPV) were read out using ImageJ software (National Institutes of Health, USA, version $1.51 \mathrm{k}$ ). According to the recommendations, ${ }^{21}$ the signal from the red channel was used for the dose reconstruction, ${ }^{2,17}$ for which maximum sensitivity is observed in the low dose region $(<1 \mathrm{~Gy}) .{ }^{4}$

At the time of Gafchromic films irradiation, the dose measurements were carried out using a reference dosimeter dedicated to fluoroscopy, i.e. an R100B detector cooperating with an RTI Barracuda electrometer. This dosimeter was calibrated in air kerma conditions (calibration certificate of 25.05.2019, expanded uncertainty $(\mathrm{k}=2) \pm 1.7 \%)$. The measuring system is shown in Figure 1. The detector indications have been corrected for the distance from the detector reference point (the active area of the reference detector was $0.35 \mathrm{~cm}$ below the detector surface, therefore the distance from the focus of the X-ray source was $30.35 \mathrm{~cm}$, the depth of the active film layer is $0.001 \mathrm{~cm}$, therefore it was assumed that the distance from the focus of the X-ray source is $30 \mathrm{~cm}$ ). In order to determine the absorbed dose (AD), the measured air kerma was multiplied by the coefficient $\mathrm{f}-$ the quotient of the mass absorption coefficients in the tissue (Gafchromic films) and air (reference dosimeter calibrated in air kerma units), $\mathrm{f}-$ factor for ICRU soft tissue $1.06^{22}$ (according to the reference for digital acquisition mode $\mathrm{f}$-factor range from $1.056(60 \mathrm{kV})$ to $1.066(95$
$\mathrm{kV})$, and from $1.058(\mathrm{HVL}=3.0 \mathrm{~mm} \mathrm{Al})$ to $1.068(\mathrm{HVL}=7.0$ $\mathrm{mm} \mathrm{Al}))^{23}$

The calibration process was as follows:

- small pieces of film $(4 \mathrm{~cm} \times 4.5 \mathrm{~cm})$ selected at random for each calibration exposure were prepared from one sheet of the selected package, assuming the maximum nonuniformity of $2 \%$ of the film sheet stated by the manufacturer (Table 1),

- the long axis of the entire film was marked on the white side of the film (in order to match the scanning direction),

- the R100B detector was placed perpendicular to the anodecathode axis of the X-ray source (to minimize the heel effect), at a distance of $30 \mathrm{~cm}$ from the focus of the X-ray source (chosen for practical reasons),

- a piece of film was placed in the reference dosimeter plane with the orange side toward the X-ray tube (the reference detector and a piece of the film were in the central area of the $\mathrm{X}$-ray beam, as seen in Figure 1), as recommended since the orange side is more sensitive and smaller error is associated with energy dependence of XR-RV3 films, ${ }^{3}$

- the treatment table was set at the Interventional Reference Point (IRP, $15 \mathrm{~cm}$ below the X-ray unit isocenter), SID (source to flat panel distance) $=100 \mathrm{~cm}$,

- the PMMA phantom $(20 \mathrm{~cm}, 30 \mathrm{~cm} \times 30 \mathrm{~cm}$ thick $)$ was placed in the X-ray beam to generate clinically used exposure conditions and also to protect the FPD (flat panel detector) of the X-ray unit against ghost effect,

- the exposure of film pieces to doses from the range of $0-8 \mathrm{~Gy}$ was performed, which gave a minimum of fifteen measuring points plus $0 \mathrm{~Gy}$ for the full range of measured doses,

- a film scanning process was carried out using Optice Pro A320 bump scanner (time $>48$ hours), each of the pieces of the film 
was placed sequentially orange side down in the central area of the scanner, ensuring minimization of the impact of scanner beam heterogeneity; as recommended in references ${ }^{4,3}$; all pieces of films were visually assessed for the presence of artifacts, possible damage, and only the pieces that passed this evaluation were included in the calibration process,

- film density readout using ImageJ software, in terms of mean pixel value (MPV) in the red channel of the image of the scanned film, size of ROI $2 \mathrm{~cm} \times 2.5 \mathrm{~cm}$, the mean value of MPV ranged from 260 to 80 with the SD from 1.1 to 2.9, i.e. standard deviations did not affect the measurement results,

- calibration curves were plotted (MPV against absorbed dose). The Gafchromic film was placed on the housing of the collimator assembly, i.e. $30 \mathrm{~cm}$ from the focus of the $\mathrm{x}$-ray source. This distance was used for practical reasons. The dose rate measured with the reference dosemeter placed next to the Gafchromic film at this distance was $216.9 \mathrm{mGy} / \mathrm{min}$ on average. Under these conditions, exposing one piece of film to a maximum of about $8 \mathrm{~Gy}$ took approximately 37 minutes. Placing the film at a distance of, for example, $70 \mathrm{~cm}$ from the focus of the x-ray source would require an exposure time of approximately $3.5 \mathrm{~h}$ in order to expose the film to the same dose value. Calibration was carried out for two film packages separately and in the dose range from 0 to nearly $8 \mathrm{~Gy}$, which was 15 exposures for the first film package and 14 for the second one. Therefore, taking into account the relatively low dose rate of ionizing radiation in the range analyzed in the study, as well as the limitation of excessive use of the x-ray apparatus, the measurements for the smallest possible distance from the x-ray tube focus were carried out.

The measurement data in the form of $\log (\mathrm{MPV})$ were plotted for the function of absorbed dose $D=f(\log M P V)$ measured during exposure using an R100B detector. Subsequently, the fitting functions were established. The analysis of the quality of the fitting functions was based on the deviation between the dose absorbed by the films during x-ray exposure (indicated by R100B detector) and the dose reconstructed from the fitting curve. The criterion of at least $95 \%$ accuracy was adopted for dose values above the dose of $50 \mathrm{mGy}$.

Third-degree polynomial as a fitting function, which was investigated in the presented study, for doses in the full range from 0 to about 8 Gy does not meet the criterion of a match above $95 \%$. Thus, measurements from doses of about 0.4 Gy to slightly above 8 Gy were isolated from the full range for the first pack of films, and measurements up to 0.6 Gy were carried out separately. For the second pack of films, two independent series of measurements were carried out (up to about 0.7 Gy and up to about $8 \mathrm{~Gy})$.

\section{X-ray unit}

Exposures of calibration films were made using the Artis zee floor X-ray unit (Siemens, max. exposure voltage $125 \mathrm{kV}$, total filtration $\geq 2.5 \mathrm{~mm} \mathrm{Al}$, additional filtration of 0.1/0.2/0.3/0.6/0.6 $\mathrm{mm} \mathrm{Cu}$ depending on the clinical program, pulsed fluoroscopy mode). In the calibration process, Coro 2020 protocol was used (voltage range: $78-78.3 \mathrm{kV}$, current range: $242.8-243.1 \mathrm{~mA}$, additional filtration $0.2 \mathrm{~mm} \mathrm{Cu}$, air kerma rate on the detector surface image: $46 \mathrm{mGy} / \mathrm{min}, 10 \mathrm{fps}$, average air kerma rate recorded by reference dosimeter $216.9 \mathrm{mGy} / \mathrm{min}, \mathrm{SD}=2.8$ $\mathrm{mGy} / \mathrm{min}$ ).

This unit is subjected to systematic quality control performed by a qualified medical physicist. All measured parameters comply with national requirements as well as with the manufacturer's recommendations. The film irradiation process was performed in clinically used conditions.

\section{Quality control of the flatbed scanner}

Gafchromic film processing was carried out in accordance with the literature recommendations using a commercially available office flatbed scanner. ${ }^{4,19}$ The scanner quality was checked by determining the heterogeneity and repeatability of the short- and long-term reading (as recommended in reference ${ }^{3}$ ). A selected sample of calibration films (irradiated with a dose of approximately $1 \mathrm{~Gy}$ ) was used for all these tests. The heterogeneity of the scanner was checked by scanning this piece of film in five positions: central and four edges, and using the relationship:

$\frac{\left|M P V_{\text {max }}-M P V_{\min }\right|}{M P V_{\text {center }}} * 100 \%$

where: $M P V_{\text {center }}$ - the value obtained when the film was positioned in the center of the scanner, $\mathrm{MPV}_{\max }, \mathrm{MPV}_{\min }-$ the maximum and minimum values obtained from scanning regardless of the position of the film in the scanner, respectively. The determined heterogeneity amounted to $0.6 \%$.

Short-term and long-term stabilities were also determined. The former was determined by scanning a selected sample of the film 10 times at intervals of several seconds, without removing the film from the scanner. The latter was determined by scanning a selected fragment of the film during each stage of scanning Gafchromic films used for patients' dose measurements (five months). The scanned area of the film was always placed in the central position of the scanner, according to the orientation established during the scanning of calibration films (long axis of the film perpendicular to the scanning direction). The repeatability was determined using the following relationship:

$\frac{\left|M P V_{\text {mean }}-M P V_{n}\right|}{M P V_{\text {mean }}} * 100 \%$

where: $\mathrm{MPV}_{\mathrm{n}}$ - value measured during a single measurement, $\mathrm{MPV}_{\text {mean }}$ - mean value from all measurements for short-term and long-term stability separately. Short-term repeatability was below $0.8 \%$, while long-term readout was below $1.1 \%$. 


\section{Results}

We analyzed polynomials from 2 nd to 5 th degree. Taking into account the practical reason (selection of the curve with the smallest degree of the polynomial that satisfies the criterion of fitting) and 95\% match criterion (compliance between the dose absorbed by the films and the dose reconstructed from the fitting curve) we chose the third-degree polynomials recommended in the literature. ${ }^{4}$ Detailed analysis performed for the selection of the best fitting function is below. The chosen criterion of $95 \%$ accuracy was based on the analysis of total uncertainty associated with the process of calibration, i.e. expanded uncertainty of radiometer, film sheet nonuniformity, stability of scanner operation, and constancy of beam quality.

The results of such analysis for the entire dose range studied showed that the differences between measurements using the reference dosimeter (R100B) during the calibration process (actual doses) and doses calculated based on the calibration function (reconstructed) are higher for an absorbed dose of the order of $50 \mathrm{mGy}(\sim 20 \%)$. For the dose range starting from about $450 \mathrm{mGy}$ these differences were below $5 \%$. After dividing the

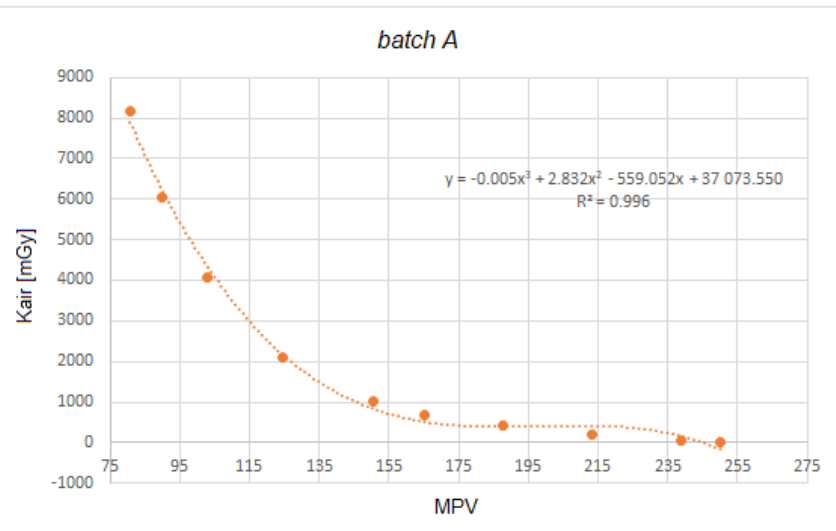

full dose range into two subranges, this difference was a maximum of $3.8 \%$ and $1.9 \%$ for the lower dose range and higher dose range, respectively. The analysis of the fit quality for the batch $A$ in the full dose range using the relation of $D=f(M P V)$ showed that the $95 \%$ match criterion was only achieved for the values of $4181.3 \mathrm{mGy}$ and higher and for $6542.5 \mathrm{mGy}$ and higher for air kerma and absorbed dose, respectively. Selected fitting curves for MPV and $\log (\mathrm{MPV})$ for a full dose range are shown in Figure 2.

For batch B of films in the full dose range, the $95 \%$ match criterion was achieved for the values of $754.9 \mathrm{mGy}$ and higher, and $800.2 \mathrm{mGy}$ and higher, for air kerma and absorbed dose, respectively. Only one point (slightly above $1 \mathrm{~Gy}$ ) did not meet the established criterion (nearly $15 \%$ deviation between the dose absorbed by the film during $\mathrm{x}$-ray exposure and the dose reconstructed from the fitting curve). Due to the identical values of deviations of the fit curves for doses within individual ranges (with the determined accuracy of polynomial presentation - up to three significant decimal numbers), detailed fitting curves were presented for the A batch of films (Figure 3).

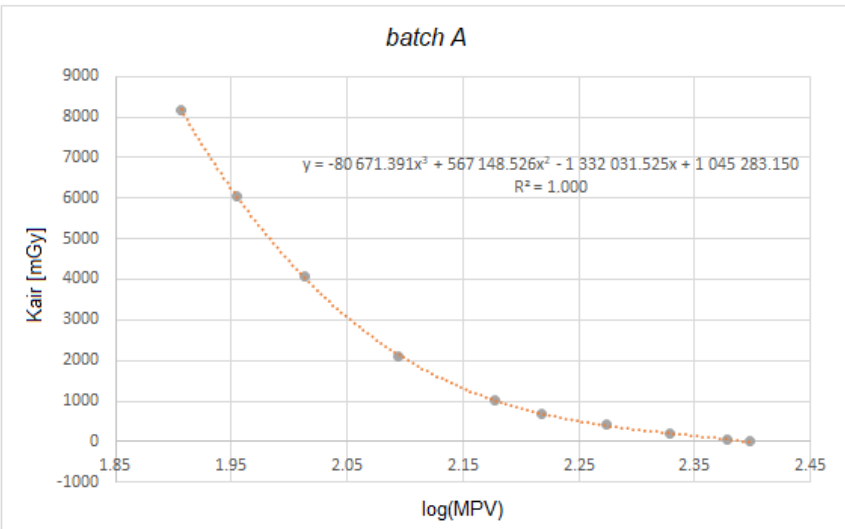

Figure 2. The fitting curve for the Gafchromic signal (MPV versus log (MPV)) for the first pack of films in the dose range from 0 to approximately 8 Gy.
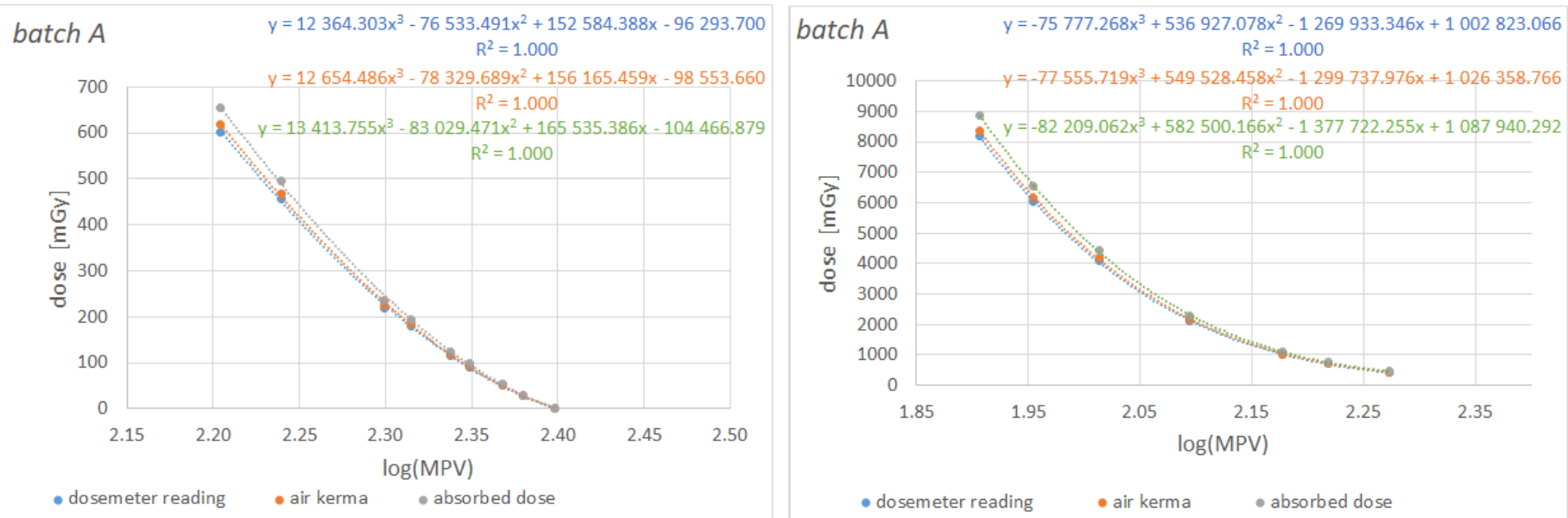

Figure 3. The fitting curve for $\log (\mathrm{MPV})$ for batch $\mathrm{A}$ of films in the lower and higher dose range, the colors of the curve and polynomial equation are matched. 

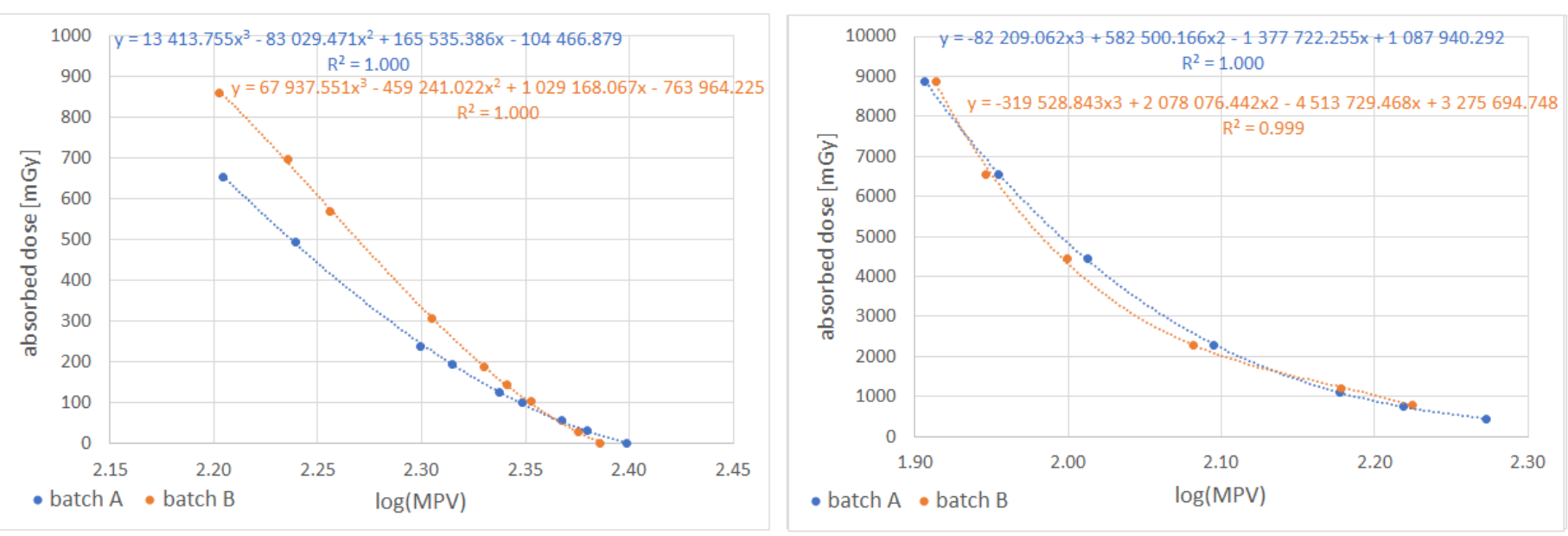

Figure 4. The fitting curve for $\mathrm{AD}=\log (\mathrm{MPV})$ for the first and second batch of films in the lower and higher dose range.

Table 2. The fitting curves for dose value with distance correction and differences in tissue and air absorption.

\begin{tabular}{cccc}
\hline \hline Batch & Absorbed dose range, AD [Gy] & 3rd-degree polynomial fit curves, AD = log (MPV) & $\left(\mathbf{R}^{2}\right)$ \\
\hline \multirow{2}{*}{$\mathbf{A}$} & $0-0.6$ & $\mathrm{y}=13413.755 \mathrm{x}^{3}-83029.471 \mathrm{x}^{2}+165535.386 \mathrm{x}-104466.879$ & 1.000 \\
& $<0.6-8.9$ & $\mathrm{y}=-82209.062 \mathrm{x}^{3}+582500.166 \mathrm{x}^{2}-1377722.255 \mathrm{x}+1087940.292$ & 1.000 \\
\hline \multirow{2}{*}{ B } & $0-0.8$ & $\mathrm{y}=67937.551 \mathrm{x}^{3}-459241.022 \mathrm{x}^{2}+1029168.067 \mathrm{x}-763964.225$ & 1.000 \\
& $<0.8-8.9$ & $\mathrm{y}=-319528.843 \mathrm{x}^{3}+2078076.442 \mathrm{x}^{2}-4513729.468 \mathrm{x}+3275694.748$ & 0.999 \\
\hline \hline
\end{tabular}

The results for the batch $\mathrm{B}$ and both dose ranges are similar to the results for batch $\mathrm{A}$. Maximum fitting deviation for the lower dose range was observed at $-4.2 \%$ for $94.19 \mathrm{mGy}$ and for the higher dose range at 4.08 for $1099.60 \mathrm{mGy}$ (the dose amount in both cases given without any correction). Figure 4 shows the curves for batch $\mathrm{B}$, as well as for batch $\mathrm{A}$ for the purposes of comparison.

The selected fitting curves and dose ranges to which they apply are presented in Table $\mathbf{2}$. Noteworthy are the high values of $\mathrm{R}^{2}$ coefficients of calibration curves determined based on $\log (\mathrm{MPV})$. For comparison, the $\mathrm{R}^{2}$ for the MPV curve was 0.996 .

The differences in dose reconstruction between the batches of Gafchromic films are presented in Figure 5. They summarize the dose reconstruction based on the signal from each batch of films and the dose reconstructed using a polynomial fit to batch A of films based on the signal from batch B (marked with an asterisk). For the $\mathrm{B}^{*}$ batch the deviation between the dose absorbed by the film during x-ray exposure and the dose reconstructed from the fitting curve ranged from 15\% (absorbed dose $102.18 \pm 1.74$ [mGy] vs. reconstructed dose $86.84 \pm 1.60$ [mGy]) to $35.9 \%(28.29 \pm 0.48[\mathrm{mGy}]$ vs. $38.45 \pm 0.44[\mathrm{mGy}])$. In the range of absorbed doses from $144 \mathrm{mGy}$ to $860 \mathrm{mGy}$ this difference ranged from $19 \%$ to $27 \%$

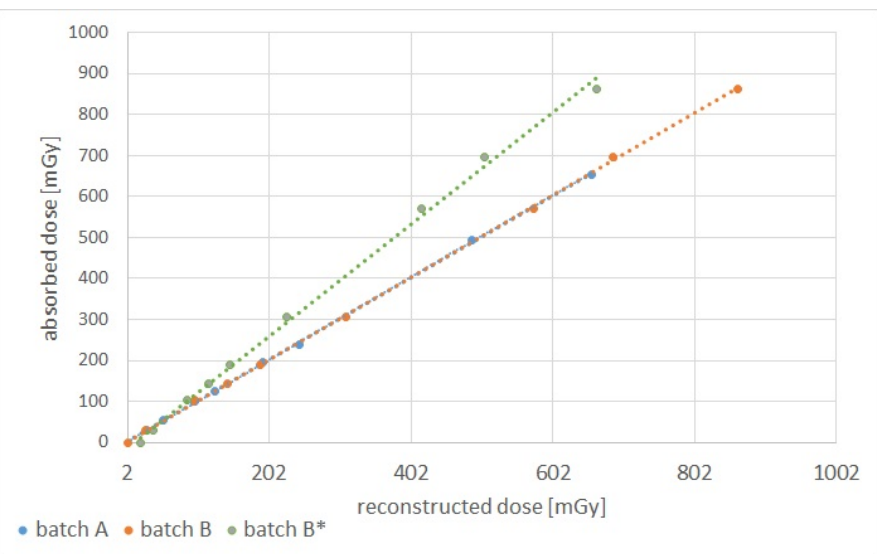

Figure 5. The differences in dose reconstruction between the batches of Gafchromic film, * - dose reconstructed by a polynomial from the batch $A$ of films based on the signal from batch $B$. 


\section{Discussion}

For type XR-RV3 Gafchromic films, there are no explicit recommendations regarding the shape of the calibration function; therefore, testing various equations of calibration curves (fit curves) available in the literature is recommended. ${ }^{4}$ Some references recommend the use of third-degree polynomials; higher polynomials are not recommended unless necessary. ${ }^{4,8}$ In choosing the appropriate form of the thirddegree polynomial of the fit curve, we analyzed the MPV and $\log$ (MPV) form curves. Only from doses of the order of 2 Gy may a calibration curve (taking MPV instead of log (MPV)) be obtained with a satisfactory fit (95\% match criterion), but not less than $92 \%$ for doses of the order of $2 \mathrm{~Gy}$. However, for higher doses, where the measured dose values were over $4 \mathrm{~Gy}$ or regardless of the correction used for doses above $6 \mathrm{~Gy}$, the accuracy of fit function was above $95 \%$. A similar result was also obtained for the second batch of films.

The necessity of calibration for each package of films is emphasized by the results presented in Figure 4. This figure shows that each series of films may have a slightly different curve, especially for the low dose range. The shape of the highdose curves is similar. For comparison, the accuracy of matching the calibration curve of the third-degree polynomial form with $\log (\mathrm{MPV})$ to the data measured in the full range (a common curve for the whole dose range) was obtained at $95 \%$ for doses from approximately $0.4 \mathrm{~Gy}$ (first pack of films) and $0.7 \mathrm{~Gy}$ (second set of films). It was concluded that the most accurate way of matching is presented by the calibration curve of the form of the third-degree polynomial with $\log (\mathrm{MPV})$ compared to the third-degree polynomial from MPV. The reasons can be seen in the fact that $\log (\mathrm{MPV})$ equals optical density, which means that one physical quantity (optical density) is associated with another physical quantity (dose). The average pixel value in the ROI cannot be called a physical quantity itself. The course of the log function means that a large change in the value of the argument of this function translates into a small change in the value of this function, which in turn makes $\log (\mathrm{x})$ less sensitive to fluctuations in the parameter $\mathrm{x}$. Therefore, it is often used where one deals with the statistical nature of the value of a given parameter, such as the interaction of ionizing radiation (e.g. film blackening) or light absorption (in our case, absorption of X-ray and scanner light when reading the films). The course of the log function somewhat narrows the range of $\log (\mathrm{x})$ values when the range of $x$ values is large, which may also affect the quality of the fit, because it affects the spread (fluctuations) of the values.

In our study, we analyzed the influence of dose range, shape of the fitting curve and its practical application on the process of determining the appropriate calibration curve of Gafchromic XR-RV3 film. The proposed method of improving the accuracy of the fit was division of calibration points into two subranges of doses, taking into account the common property of radiochromic films of nonlinearity of optical density gaining as a consequence of exposure to low and high doses of ionizing radiation. In our study, one fitting curve for doses in the full range from 0 to about 8 Gy does not meet our criterion (above 95\% compliance between the dose absorbed by the films and the dose reconstructed from the fitting curve).

All measurement series contain a 0 Gy point (MPV reading of the non-exposed film of the given film packet). Dose ranges were called lower (nine calibration points) and higher dose ranges (seven calibration points), respectively. The increased number of measuring points in the range up to $1 \mathrm{~Gy}$ in relation to the entire range of doses of calibration films is also recommended in the literature, which suggests a significant impact of the number of measuring points for doses up to $1 \mathrm{~Gy}$ on the results obtained. ${ }^{4}$ Extrapolation of the calibration curve is not recommended for doses outside the calibration points, ${ }^{4}$ therefore, from a practical point of view the endpoint has been set at a value close to $8 \mathrm{~Gy}$.

We also showed that for doses up to $100 \mathrm{mGy}$ in the low dose range and for doses up to $1000 \mathrm{mGy}$ in the high dose range, the curves for the value read from the dosemeter, air kerma and the absorbed dose are the same, i.e. curves in these ranges overlap as shown in Figure 3.

The differences in dose reconstruction between the batches of Gafchromic film were also studied. We compared the dose reconstructed by a polynomial from the first batch of films based on the signal from the second batch with those received in accordance with the recommendations (dose reconstructed by a polynomial from the given batch of films based on the signal from the same batch). The deviation was observed at the level up to $36 \%$ compared to about $7 \%$ (assumed to be adequate for normal tissue complication risk). ${ }^{24}$

According to literature information, the accuracy of dose measurement using Gafchromic XR-RV3 films is estimated at $20 \%(\mathrm{k}=1)^{1,3,4,14}$ and $13.2 \%(\mathrm{k}=1) .^{25}$ This accuracy depends primarily on fit curves, beam quality $(2.0 \%),{ }^{4,25}$ film uniformity, quality of the scanner (stability and uniformity) and dose measurement. ${ }^{1,4}$ In our study the film's nonuniformity within one batch was up to $2 \%$. We also showed that short-term repeatability of the scanner was below $0.8 \%$, long-term readout stability was up to $1.1 \%$ and determined heterogeneity amounted to $0.6 \%$. The expanded uncertainty for dose measurement with R100B dosimeter was $\pm 1.7 \%$ ( $\mathrm{k}=2$ ). Taking into account the recommendations from the literature regarding the fitting function, we chose a third-order polynomial. This kind of function ensured the quality of the fit, while the results showed that the exponential fits could not correctly reproduce the XRRV3 response. ${ }^{4}$ In the whole dose range we used fifteen measurement points plus the $0 \mathrm{~Gy}$ point to reliably reproduce the doses measured against those calculated on the basis of the calibration curve while the literature recommends eight (evenly spaced) calibration points to achieve an optimal, satisfactory calibration curve. $^{4}$ 


\section{Conclusions}

The advantage of the calibration curve in the form of $\log (\mathrm{MPV})$ is the functionality in the low dose range, where dose reconstruction accuracy could be maintained at a high $95 \%$ level. To evaluate the robustness of the calibration fitting the 95\% criterion of a match between the dose absorbed by the Gafchromic films during $x$-ray exposure and the dose reconstructed from the fitting curve was used. For better low dose evaluation and for 95\% compliance between the dose absorbed by the films and the dose reconstructed from the fitting curve splitting the dose range for two ranges and increased number of measuring points in the range up to $1 \mathrm{~Gy}$ in relation to the entire dose range was done.

Using 3rd order polynomial as a calibration fitting function, the level of $95 \%$ accuracy could be achieved by splitting the dose range seen in interventional radiology (up to 8Gy) into low- and high-dose subranges.

Other polynomials than 3rd order are not practical to use during calibration of XR-RV3 Gafchromic films due to lower R2 values, which results in less accurate dose reconstruction.
Gafchromic films from different batches may not be as similar as one could expect, due to material inhomogeneity. Therefore, using the calibration curve obtained on the base of one film packet to the dose reconstruction from another film batch, one may introduce the systematic error of even $20 \%$ as it was demonstrated.

\section{Disclosure of interest}

The authors declare that they have no conflicts of interest concerning this article.

\section{Acknowledgements}

This research was funded by the statutory funds of the Medical University of Silesia and by an unrestricted grant from the Elektrokardiologia-Ochojec Foundation in Katowice, NGO KRS 0000296037.

\section{References}

1. Farah J, Trianni A, Carinou E, et al. Measurement of maximum skin dose in interventional radiology and cardiology and challenges in the set-up of European alert thresholds. Radiat Prot Dosimetry. 2015;164(1-2):138-142. https://doi.org/10.1093/rpd/ncu314

2. Greffier J, Van Ngoc Ty C, Agelou M, et al. Impact of the calibration conditions of XR-RV3 films on peak skin dose measurements in interventional radiology. Radiat Prot Dosimetry. 2017;174(2):207-215. https://doi.org/10.1093/rpd/ncw116

3. McCabe BP, Speidel MA, Pike TL, Van Lysel MS. Calibration of GafChromic XR-RV3 radiochromic film for skin dose measurement using standardized x-ray spectra and a commercial flatbed scanner. Med Phys. 2011;38(4):1919-1930. https://doi.org/10.1118/1.3560422

4. Farah J, Trianni A, Ciraj-Bjelac O, et al. Characterization of XR-RV3 GafChromic(®) films in standard laboratory and in clinical conditions and means to evaluate uncertainties and reduce errors. Med Phys. 2015;42(7):4211-4226. https://doi.org/10.1118/1.4922132

5. Wen N, Lu S, Kim J, et al. Precise film dosimetry for stereotactic radiosurgery and stereotactic body radiotherapy quality assurance using GafchromicTM EBT3 films. Radiat Oncol. 2016;11(1):1-11. https://doi.org/10.1186/s13014-016-0709-4

6. Girardi A, Anglesio S, Amadore G, Trevisiol E, Redda MG. Implementation of a program for quality assurance on leaf positioning accuracy using Gafchromic ® RTQA2 films. J Med Phys. 2014;39(2):116-120. https://doi.org/10.4103/0971-6203.131287

7. León Marroquin EY, Herrera González JA, Camacho López MA, Villarreal Barajas JE, García-Garduño OA. Evaluation of the uncertainty in an EBT3 film dosimetry system utilizing net optical density. J Appl Clin Med Phys. 2016;17(5):466-481. https://doi.org/10.1120/jacmp.v17i5.6262

8. Paelinck L, De Neve W, De Wagter C. Precautions and strategies in using a commercial flatbed scanner for radiochromic film dosimetry. Phys Med Biol. 2007;52(1):231-242. https://doi.org/10.1088/0031-9155/52/1/015

9. Miller DL, Balter S, Cole PE, et al. Radiation doses in interventional radiology procedures: the RAD-IR study: part I: overall measures of dose. J Vasc Interv Radiol. 2003;14(6):711-727. https://doi.org/10.1097/01.rvi.0000079980.80153.4b

10. Costa KC, Gomez AML, Alonso TC, Mourao AP. Radiochromic film calibration for the RQT9 quality beam. Radiat Phys Chem. 2017;140:370-372. https://doi.org/https://doi.org/10.1016/j.radphyschem.2017.02.032

11. Bin JH, Ji Q, Seidl PA, et al. Absolute calibration of GafChromic film for very high flux laser driven ion beams. Rev Sci Instrum. 2019;90(5):053301. https://doi.org/10.1063/1.5086822

12. Gorny KR, Leitzen SL, Bruesewitz MR, Kofler JM, Hangiandreou NJ, McCollough CH. The calibration of experimental selfdeveloping Gafchromic ${ }^{\circledR}$ HXR film for the measurement of radiation dose in computed tomography. Med Phys. 2005;32(4):10101016. https://doi.org/https://doi.org/10.1118/1.1862802

13. Tomic N, Quintero C, Whiting BR, et al. Characterization of calibration curves and energy dependence GafChromicTM XR-QA2 model based radiochromic film dosimetry system. Med Phys. 2014;41(6Part1):62105. https://doi.org/https://doi.org/10.1118/1.4876295 
14. Greffier J, Goupil J, Larbi A, et al. Assessment of patient's peak skin dose during abdominopelvic embolization using radiochromic (Gafchromic) films. Diagn Interv Imaging. 2018;99(5):321-329. https://doi.org/10.1016/j.diii.2017.12.008

15. Balter S. Methods for measuring fluoroscopic skin dose. Pediatr Radiol. 2006;36:136-140. https://doi.org/10.1007/s00247-006-01933

16. Chaikh A, Gaudu A, Balosso J. Monitoring methods for skin dose in interventional radiology. Int Journalof Cancer Ther Oncol. 2014;3:3011. https://doi.org/10.14319/ijcto.0301.1

17. Greffier J, Van Ngoc Ty C, Agelou M, et al. Impact of the calibration conditions of XR-RV3 films on peak skin dose measurements in interventional radiology. Radiat Prot Dosimetry. 2017;174(2):207-215. https://doi.org/10.1093/rpd/ncw116

18. Devic S, Tomic N, Lewis D. Reference radiochromic film dosimetry: Review of technical aspects. Phys Medica. 2016;32(4):541-556. https://doi.org/10.1016/j.ejmp.2016.02.008

19. Setilo I, Du Plessis FCP. Dosimetric comparison between XR-RV3 and EBT2 radiochromic film in megavoltage photon beams. Int J Radiat Res. 2016;14(2):149-152. https://doi.org/10.18869/acadpub.ijrr.14.2.149

20. Pasquino M, Cutaia C, Poli M, et al. Patient's Peak Skin Dose evaluation using Gafchromic films in interventional cardiology procedures and its correlation with other dose indicators. Phys Medica. 2018;53:103-107. https://doi.org/10.1016/j.ejmp.2018.08.017

21. Niroomand-Rad A, Chiu-Tsao S-T, Grams MP, et al. Report of AAPM Task Group 235 Radiochromic Film Dosimetry: An Update to TG-55. Med Phys. 2020;47(12):5986-6025. https://doi.org/https://doi.org/10.1002/mp.14497

22. Jones AK, Ensor JE, Pasciak AS. How accurately can the peak skin dose in fluoroscopy be determined using indirect dose metrics? Med Phys. 2014;41(7):71913. https://doi.org/10.1118/1.4884020

23. Jones AK, Pasciak AS. Calculating the peak skin dose resulting from fluoroscopically guided interventions. Part I: Methods. J Appl Clin Med Phys. 2011;12(4):3670. https://doi.org/10.1120/jacmp.v12i4.3670

24. Thwaites D. Accuracy required and achievable in radiotherapy dosimetry: have modern technology and techniques changed our views? J Phys Conf Ser. 2013;444:12006. https://doi.org/10.1088/1742-6596/444/1/012006

25. Greffier J, Grussenmeyer-Mary N, Larbi A, et al. Experimental evaluation of a radiation dose management system-integrated 3D skin dose map by comparison with XR-RV3 Gafchromic ${ }^{\circledR}$ films. Phys Medica. 2019;66:77-87. https://doi.org/10.1016/j.ejmp.2019.09.234 